\title{
Toxicidade de materiais de construção: uma questão incontornável na construção sustentável
}

\author{
Toxicity of building materials: a key issue in sustainable \\ construction
}

\begin{tabular}{|c|c|}
\hline & $\begin{array}{l}\text { Fernando Pacheco Torgal } \\
\text { Said Jalali }\end{array}$ \\
\hline & Resumo \\
\hline $\begin{array}{l}\text { Fernando Pacheco Torgal } \\
\text { Unidade de Investigação C-TAC- }\end{array}$ & $\begin{array}{l}\text { vitar o uso de materiais de construção tóxicos é um dos princípios da } \\
\text { construção sustentável. Os edifícios de habitação contêm muitos } \\
\text { materiais tóxicos, alguns deles mesmo em conformidade com } \\
\text { normativos regulamentares aplicáveis na Europa e em Portugal. Parte }\end{array}$ \\
\hline $\begin{array}{r}\text { Grupo Construção Sustentável } \\
\text { Universidade do Minho }\end{array}$ & Palavras-chave: Toxicidade. COVs. Amianto. Canalizacão em chumbo. \\
\hline & 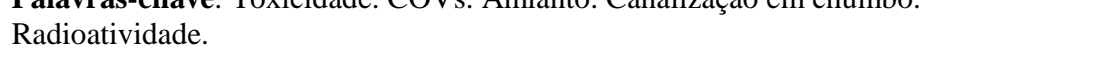 \\
\hline $\begin{array}{r}\text { CEP: } 4800-058 \\
\text { Tel.:+351 253-510200 }\end{array}$ & Abstract \\
\hline $\begin{array}{r}\text { E-mail: } \\
\text { f.pachecotorgal@gmail.com }\end{array}$ & $\begin{array}{l}\text { Avoiding the use of toxic building materials is one of the principles of sustainable } \\
\text { construction. Current residential buildings frequently contain many toxic }\end{array}$ \\
\hline $\begin{array}{r}\text { Said Jalali } \\
\text { Departamento de Engenharia } \\
\text { Civil } \\
\text { Campus de Azurém }\end{array}$ & $\begin{array}{l}\text { materials, some of which are even compliant with legal regulations in Europe and } \\
\text { Portugal. Part of the problem is due to the fact that architects and civil engineers } \\
\text { receive no education on the toxicity of building materials. Another problem is }\end{array}$ \\
\hline & $\begin{array}{l}\text { related to the fact that the regulations about toxicity thresholds are influenced by } \\
\text { economic issues. This paper discusses some cases of toxic building materials by }\end{array}$ \\
\hline $\begin{array}{r}\text { Tel.:+351 253-510200 } \\
\text { Universidade do Minho } \\
\text { E-mail: said@civil.uminho.pt }\end{array}$ & $\begin{array}{l}\text { reviewing previously published work. It is focused on the emission of volatile } \\
\text { organic compounds (VOCs); the toxicity of impregnating agents, paints, varnishes } \\
\text { and other building materials; materials that release toxic fumes when on fire; }\end{array}$ \\
\hline Recebido em 09/04/2010 & asbestos-based materials; radioactive materials; and lead plumbing. \\
\hline Aceito em 28/07/2010 & Toxicity. VOCs. Asbestos. Lead plumbing. Radioactivity. \\
\hline
\end{tabular}




\section{Introdução}

Enquanto a maioria das construções até ao início do século $\mathrm{XX}$ era feitas de materiais naturais, as construções correntes executadas com materiais produzidos industrialmente podem conter substâncias químicas como metais pesados e COV. Estes compostos podem provocar inúmeros problemas de saúde, nomeadamente os seguintes: irritações da pele, olhos e vias respiratórias; distúrbios cardíacos, digestivos, renais ou hepáticos; dores de cabeça e mal-estar generalizado; distúrbios do sistema nervoso, como perturbações da memória, de atenção, concentração e da fala, estresse e ansiedade; perturbações do sistema hormonal (problemas fetais e de reprodução); e desenvolvimento de cancros das fossas nasais, dos seios frontais e pulmões, quando presentes em elevadas concentrações. Para lá da exposição direta e diária decorrente da presença no interior das habitações de materiais com algum grau de toxicidade, importa não esquecer o elevado potencial de periculosidade que está associado à produção de produtos químicos, bem patente no desastre de Bopal, ocorrido na Índia em 1984, quando um complexo industrial de produtos químicos libertou uma nuvem de isocianato de metilo que provocou aproximadamente 15.000 mortos e problemas de saúde em quase 200.000 pessoas (SATYANAND, 2008; VARMA; MULAY, 2006). Durante a fase de produção de alguns materiais de construção, há emissão de vários tipos de poluentes, assim como também há lugar à produção de resíduos muito perigosos, os quais irão afetar negativamente e de alguma forma o ambiente do planeta onde vivemos, sendo obrigatório que os referidos impactos sejam assacados aos materiais em questão. Desses poluentes merecem destaque os descritos a seguir.

Organoclorados (dioxinas e furanos): as dioxinas e os furanos são resíduos químicos provenientes de processos industriais que envolvem cloro, como os processos relacionados com a produção de PVC. São compostos extremamente tóxicos para a saúde (INTERNATIONAL AGENCY FOR RESEARCH ON CANCER, 1997; KOOPMAN-ESSEBOOM $e t$ al., 1996; LANTING et al., 1998), com a agravante de serem bioacumuláveis no organismo. Essa periculosidade estende-se por óbvias razões à biodiversidade por via da contaminação de toda a cadeia alimentar (OPPENHUIZEN; SIJM, 1990; TILLITT et al., 1993). Análises químicas realizadas em golfinhos do Pacífico Norte revelaram concentrações de dioxinas entre 13 e 37 milhões de vezes superiores à concentração das mesmas dioxinas na água do mar dessa região
(THORTON, 2002). Vários grupos de cientistas sugeriram já a proibição da utilização de cloro como matéria-prima industrial (FLORES et al., 2004).

Ftalatos: são grupos de compostos químicos derivados do ácido ftálico, utilizado como aditivo para reduzir a rigidez dos materiais plásticos. O caso mais conhecido de materiais de construção que utilizam ftalatos na sua composição diz respeito ao PVC, tanto em caixilharias como em canalizações para águas e esgotos. São vários os estudos que comprovam a toxicidade desses compostos para a saúde humana (LOVEKAMPSWAN; DAVIS, 2003; HAUSER; CALAFAT, 2005; HEUDORF et al., 2007; WOLFF et al., 2008; SWAN, 2008; MEEKER et al., 2009). A toxicidade faz-se sentir não só ao nível da contaminação de toda a cadeia alimentar devido aos resíduos gerados na produção de PVC, mas também na ingestão de água a partir de canalizações de PVC.

Compostos orgânicos voláteis (COVs): são poluentes atmosféricos libertados por materiais de construção contendo solventes orgânicos como tintas, vernizes, colas e selantes. A redução da ventilação no interior das habitações para se minimizarem gastos energéticos contribui para aumentar o volume desses poluentes e para agravar os seus efeitos sobre a saúde (HANSEN; BURROUGHS, 1999; SAMFIELD, 1992; STERLING, 1985). Além disso, os COVs contribuem para a formação de ozônio troposférico, um gás que provoca efeito de estufa.

Para além dos materiais responsáveis pela produção de dioxinas, furanos, ftalatos e COVs, no presente capítulo são ainda abordados os materiais que libertam fumos tóxicos em caso de incêndio, os materiais contendo substâncias radioativas, as situações de toxicidade relacionadas à presença de amianto em materiais de construção e também ao chumbo em canalizações de abastecimento de água. O enquadramento regulamentar analisado diz respeito ao espaço europeu e, mais particularmente, a Portugal.

\section{Emissão de COVS para tintas e vernizes}

Para além da libertação de COVs, provocada pelas tintas e vernizes, que é nefasta para a saúde (KOSTIAINEN, 1995) e para o meio ambiente, esses materiais contêm ainda metais pesados com elevado poder cancerígeno (Quadro 1). 


\begin{tabular}{|l|l|}
\hline \multicolumn{1}{|c|}{ Agente cancerígeno } & \multicolumn{1}{c|}{ Fonte } \\
\hline Cromo & Primários, tintas \\
\hline Cádmio & Pigmentos \\
\hline Benzeno & Solventes \\
\hline Cloreto de metileno & Decapantes \\
\hline Estireno & Solventes orgânicos \\
\hline Níquel & Pigmentos \\
\hline Chumbo & Primários, secantes, pigmentos \\
\hline
\end{tabular}

Fonte: International Agency for Research on Cancer (1995) e United Nations Centre for Human Settlements (1997). Quadro 1 - Agentes com poder cancerígeno presentes em tintas

Outros autores (KWOK et al., 2003) confirmam a emissão de COVs em materiais com acabamentos em verniz. Recentemente, Salasar (2007) analisou as emissões de COV em tintas à base de solventes orgânicos e à base de água, tendo concluído que as primeiras chegam a emitir 520 vezes mais COVs que as segundas. Em termos legislativos o Decreto-lei $\mathrm{n}^{\mathrm{o}} 181 / 2006$, de 6 de setembro, procedeu à transposição para a ordem jurídica portuguesa a Diretiva $n^{\circ}$ 204/42/CE, de 21 de abril de 2004, que limita o teor de COVs em tintas e vernizes (Quadro 2). Contudo, somente em julho de 2007 é que o Ministério do Ambiente, por meio do Despacho $\mathrm{n}^{\circ}$ 17.141/2007, aprovou um programa para controle da aplicação do referido decreto-lei, o que permite que se tenha uma ideia da quantidade de materiais já aplicados no setor da construção que contêm teores de COVs muito superiores aos novos limites.

\section{Toxicidade de produtos de impregnação de madeiras}

Embora sendo um material de excelência para uma construção mais sustentável, a madeira padece de baixa resistência à degradação por agentes biológicos, fungos e insetos (carunchos e térmitas). Enquanto os fungos e térmitas degradam a madeira aplicada em locais úmidos, os carunchos costumam atacar madeiras com teores de umidade correntes no interior das habitações (CRUZ; NUNES, 2009). Até muito recentemente a preservação das madeiras implicava a sua impregnação com inseticidas ou fungicidas, produtos como o creosote ou outros à base de sais metálicos como cobre, cromo e arsênico (CCA). Os referidos sais metálicos são bastante tóxicos, além de ser bioacumuláveis. Quando em contacto com a água da chuva ou outra, grande parte desses sais acaba sendo lixiviada, contaminando o meio ambiente. Desde $1^{\circ}$ de janeiro de 2004 a Agência de Protecção Ambiental dos Estados Unidos (EPA) proibiu o uso de CCA no tratamento de madeiras para fins habitacionais (EDLICH;
WINTERS; LONG, 2005). O creosote contém agentes de elevado potencial cancerígeno (ATSDR, 2002; SMITH, 2008), pelo que desde 2001 a Directiva da União Europeia 2001/90/EC iniciou um processo progressivo que visa à proibição do uso de creosote no tratamento de madeiras. Estudos recentes (THIERFELDER; SANDSTROM, 2008) referem que muitas das travessas utilizadas nos caminhos de ferro apresentam elevado teor de creosote, superior mesmo aos limites definidos pela regulação europeia, pelo que passam a ser considerados resíduos perigosos, o que condiciona o seu depósito e tratamento em fim de vida (PRUSZINSKI, 1999). Considerações similares podem e devem ser feitas no que se refere aos resíduos de construção e demolição que contenham madeiras impregnadas com materiais tóxicos.

\section{Materiais que libertam fumos tóxicos em caso de incêndio}

Outra situação de toxicidade dos materiais de construção prende-se com a libertação de fumos e substâncias tóxicas em caso de incêndio. Alguns estudos apontam mesmo para o fato de a maioria das mortes em caso de incêndio estar precisamente relacionada com a inalação de gases tóxicos, e também de esse número ter vindo a aumentar desde o fim da década de 80 , o que pode estar relacionado com o aumento no interior das habitações de materiais mais combustíveis e mais tóxicos em caso de incêndio (GANN et al., 1994; HALL; HARWOOD, 1995; WU, 2001; LEVIN; KULIGOWSKI, 2005). Liang e Ho (2007) analisaram a toxicidade após a combustão de vários isolantes térmicos e concluíram que tanto o polietileno como o poliuretano apresentam elevada toxicidade, pois excedem o valor limite de 10 , correspondente a materiais de baixa toxicidade em caso de incêndio (Figura 1). Esse índice é obtido a partir da análise das emissões de 14 tipos de gases de combustão para uma concentração-base que seja fatal ao fim de $30 \mathrm{~min}$. Esses autores 
recomendam que a utilização de poliuretano ou polietileno só possa fazer-se se eles forem protegidos por outros materiais incombustíveis. Doroudiani e Omidian (2010) recomendam a não utilização de molduras decorativas de poliestireno, devido à sua elevada combustibilidade e à libertação de fumos tóxicos em caso de incêndio. Estes autores referem que, embora esse material tenha vindo recentemente a ser produzido com um aditivo retardador de ignição, em caso de incêndio, ele gera brometo de hidrogênio, um gás tóxico.

\begin{tabular}{|c|c|c|c|}
\hline Subcategoria de produtos & Tipos & $\begin{array}{l}\text { (g/l) a partir de } 1^{\circ} \\
\text { de janeiro de } 2007\end{array}$ & $\begin{array}{l}\text { (g/l) a partir de } 1^{\circ} \\
\text { de janeiro de } 2010\end{array}$ \\
\hline \multirow{2}{*}{ a) Tintas mate* para paredes e tetos interiores } & $\mathrm{BA}$ & 75 & 30 \\
\hline & BS & 400 & 30 \\
\hline \multirow{2}{*}{ b) Tintas brilhantes para paredes e tetos interiores } & BA & 150 & 100 \\
\hline & BS & 400 & 100 \\
\hline \multirow{2}{*}{ c) Tintas para paredes exteriores de substrato mineral } & BA & 75 & 40 \\
\hline & BS & 450 & 30 \\
\hline \multirow{2}{*}{$\begin{array}{l}\text { d) Tintas para remates e painéis interiores/exteriores } \\
\text { de madeira ou metal }\end{array}$} & BA & 150 & 130 \\
\hline & BS & 400 & 300 \\
\hline \multirow{2}{*}{$\begin{array}{l}\text { e) Vernizes e lasures } * * \text { para remates } \\
\text { interiores/exteriores, incluindo lasures opacas }\end{array}$} & BA & 150 & 130 \\
\hline & BS & 500 & 400 \\
\hline \multirow{2}{*}{$\begin{array}{l}\text { f) Lasures com poder de enchimento para interiores e } \\
\text { exteriores }\end{array}$} & BA & 150 & 130 \\
\hline & BS & 700 & 700 \\
\hline \multirow{2}{*}{ g) Primários } & BA & 50 & 30 \\
\hline & BS & 450 & 350 \\
\hline \multirow{2}{*}{ h) Primários fixadores } & $\mathrm{BA}$ & 50 & 30 \\
\hline & BS & 750 & 750 \\
\hline \multirow{2}{*}{$\begin{array}{l}\text { i) Produtos de revestimento de alto desempenho } \\
\text { monocomponente }\end{array}$} & BA & 140 & 140 \\
\hline & BS & 600 & 500 \\
\hline \multirow{2}{*}{$\begin{array}{l}\text { j) Produtos de revestimentos reativos de alto } \\
\text { desempenho bicomponente para utilizações finais } \\
\text { específicas, nomeadamente em pisos }\end{array}$} & BA & 140 & 140 \\
\hline & BS & 550 & 500 \\
\hline \multirow{2}{*}{ k) Produtos de revestimento multicolor } & $\mathrm{BA}$ & 150 & 100 \\
\hline & BS & 400 & 100 \\
\hline \multirow{2}{*}{ 1) Produtos de revestimento de efeito decorativo } & BA & 300 & 200 \\
\hline & BS & 500 & 200 \\
\hline
\end{tabular}

* Fosca é o termo utilizado no Brasil

** Impregnante é a designação utilizada no Brasil

Legenda:

BA: Tintas com viscosidade ajustada por água; e

BS: Tintas com viscosidade ajustada por solventes orgânicos

Quadro 2 - Teor máximo de COVs para tintas decorativas e vernizes nos termos do Decreto-lei $\mathrm{n}^{\circ}$ 181/2006, de 6 de setembro (PORTUGAL, 2006) 


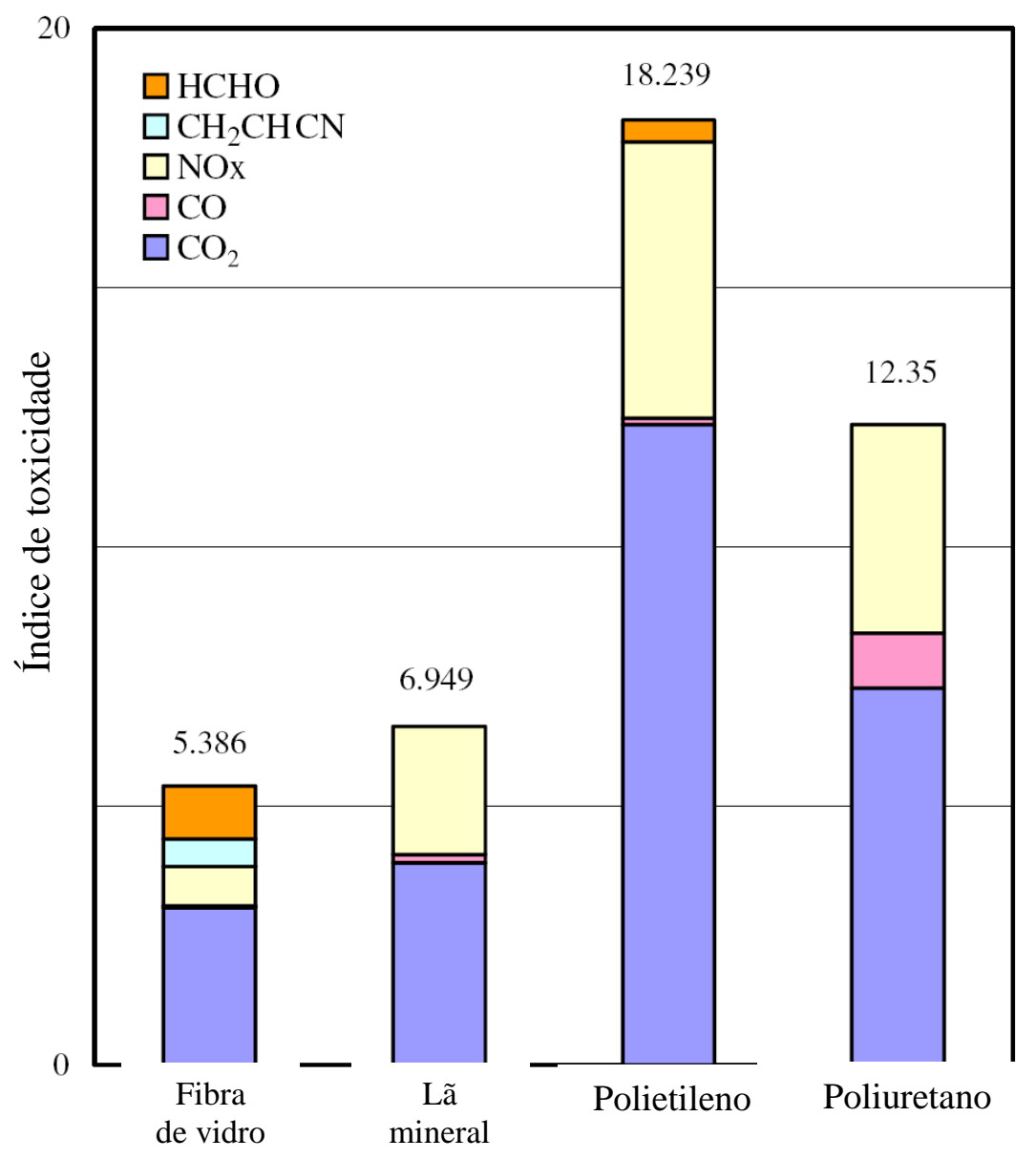

Fonte: Liang e Ho (2007).

Figura 1 - Valor médio do índice de toxicidade para vários isolamentos térmicos

\section{Materiais contendo amianto}

Nos termos do Decreto-lei no 266/2007, de 24 de julho, o amianto compreende as fibras minerais com um comprimento de $5 \mu \mathrm{m}$ e diâmetro inferior a $3 \mu \mathrm{m}$, do grupo da serpentina (crisólito) ou do grupo das anfibolas (actinolite, grunerite/amosite, antofilite, arocidolite e tremolite). Estas fibras são também designadas por "asbestos", que advêm da designação do grego para um material incombustível. Essa propriedade, aliada a uma elevada resistência à tração, facilidade para ser tecida e baixo custo, entre outras, levou a que o uso dessas fibras rapidamente se vulgarizasse ao nível da indústria da construção, quer como isolante térmico e antifogo, quer principalmente na produção de painéis de fibrocimento. A partir da década de 60 vários estudos concluíram pela relação entre o aparecimento de várias doenças profissionais e a exposição ao amianto. $\mathrm{Na}$ altura considerou-se que só algumas fibras minerais produziam efeitos nefastos para a saúde, pelo desenvolvimento de mesotelioma (cancro do revestimento mesotelial do pulmão relativamente ao qual a maior parte dos doentes morre em menos de 12 meses após o diagnóstico) (AZUMA et al., 2009; BIANCHI et al., 1997; JARVHOLM; ENGLUND; ALBIN, 1999), razão pela qual esse material continuou a ser utilizado. Somente na década de 80, com a aprovação da Diretiva 83/477/CEE, que definia os riscos para a saúde dos trabalhadores expostos ao amianto, é que a periculosidade daquele material começou a ser levada efetivamente a sério. Em 1991 uma nova diretiva (91/382/EEC) agravou os limites previstos na diretiva inicial. Portugal, só passados seis anos, é que introduziu no seu Direito interno essas preocupações, por meio do Decreto-lei no 284/89, de 24 de agosto. As investigações, entretanto, feitas pela comunidade científica permitiram concluir que todas as fibras de amianto apresentavam potencial cancerígeno, na variante de asbestose, lesões do tecido pulmonar causadas por um ácido produzido pelo organismo na tentativa de dissolver as fibras (AKIRA, 2010), ou mesmo de cancros do pulmão, do trato 
gastrointestinal, dos rins e da laringe (ANTONESCU-TURCU; SCHAPIRA, 2010; LADOU, 2004; SILVERSTEIN WELCH; LEMEN, 2009).

Consequentemente, a Diretiva 2003/18/EC veio proibir a extração dessas fibras e a sua utilização em produtos. Portugal transpôs esta diretiva para o seu Direito interno através do Decreto-lei $\mathrm{n}^{\circ}$ 266/2007, de 24 de julho, o qual define que existe risco para a saúde quando há exposição dos trabalhadores a ambientes com fibras superiores ao limite de exposição (VLE) de 0,1 fibra por centímetro cúbico. Muito embora se possa pensar que o amianto deixou de constituir um problema desde que foi proibida a sua produção no espaço da União Europeia, a verdade é que só em Portugal existem 600.000 hectares de coberturas de fibrocimento contendo amianto. E se é verdade que a periculosidade dessa espécie particular de aplicação seja menor pelo fato de as fibras estarem embebidas na matriz da pasta de cimento, também é verdade que eventuais quebras dessas coberturas propiciarão a libertação das fibras de amianto. Isso já para não referir que os produtos de hidratação do cimento se degradam ao longo do tempo, pelo que a probabilidade de libertação de fibras aumentará com o tempo. Não há assim qualquer garantia de que os ocupantes de edifícios com coberturas de fibrocimento não estejam expostos a um valor superior ao limite de exposição (VLE), conforme definido no art. $4^{\circ}$ do Decreto-lei $n^{\circ}$ 266/2007, de 24 de julho, nem tampouco se exposições ainda que para valores inferiores ao VLE não poderão resultar em graves problemas de saúde no longo prazo

Nota-se que, segundo a OMS, não são conhecidos limites de exposição abaixo dos quais se pode garantir que não há risco cancerígeno. Em situação bastante mais grave estão os casos de edifícios ou pavilhões industriais em que o amianto foi utilizado por projeção na sua forma friável, mas cuja remoção só pode ser feita por firmas especializadas, como prevê o Decreto-lei $n^{0}$ 266/2007, de 24 de julho. Contudo, sendo esse instrumento jurídico muito recente, parece evidente que só daqui a vários anos as preocupações consignadas nele serão levadas em conta pelas entidades com responsabilidades formativas, significando isso que os técnicos que recentemente se diplomaram na área da Engenharia Civil pouco ou nada ouviram falar a esse respeito, e só daqui a vários anos podemos esperar ter técnicos que no âmbito de unidades curriculares de materiais de construção e outras estejam sensibilizados e preparados para lidar com esse problema.

\section{Materiais radioativos}

A utilização de materiais com resíduos com algum tipo de contaminação radiológica é reconhecida por vários autores como algo que deve merecer preocupação em termos de periculosidade para a saúde pública, já que a exposição mesmo a baixas doses de radiação por longos períodos pode resultar no desenvolvimento de cancros (INTERNATIONAL COMMISSION ON RADIOLOGICAL PROTECTION, 1990). Regra geral, a maioria dos materiais de construção não apresenta níveis de radiação preocupantes (PAPAEFTHYMIOU; GOUSETI, 2008), o que já não se sucede com alguns subprodutos utilizados principalmente no fabrico de betão, como o fosfogesso, as escórias de alto-forno e algumas cinzas volantes (Tabela 1). Alguns tipos de fosfogesso contêm metais pesados e elementos radioativos, como rádio $\left({ }^{226} \mathrm{Ra}\right)$, chumbo $\left({ }^{210} \mathrm{~Pb}\right) \mathrm{e}$ urânio $\left({ }^{238} \mathrm{U},{ }^{234} \mathrm{U}\right)$, provenientes das rochas fosfatadas (RIANEK, 1971). O uso de fosfogesso que exceda um nível de radioatividade de 370 $\mathrm{Bq} / \mathrm{kg}$ (em que $1 \mathrm{~Bq}$ corresponde a 1 desintegração nuclear por segundo) encontra-se proibido desde 1992 (UNITED STATES ENVIRONMENTAL PROTECTION AGENCY, 1992). Já o limite proposto pela European Atomic Energy Community (1996) é de $500 \mathrm{~Bq} / \mathrm{kg}$.

Canut (2006) refere o fato de as rochas fosfáticas brasileiras apresentarem baixo nível de radioatividade natural, pelo que o fosfogesso produzido naquele país apresenta valores de radioatividade abaixo do valor-limite fixado pela United States Environment Protection Agency (EPA). Outro aspecto que deve ser acautelado tem que ver com o gás radão, ${ }^{1}$ que se encontra associado ao elemento rádio, presente em alguns tipos de fosfogesso e que tenderá a ser particularmente nefasto para materiais de construção à base de fosfogesso colocados em ambientes de baixa ventilação (KOVLER, 2009).

O radão é um gás de origem natural, radioativo, cujos átomos se desintegram originando outros elementos também radioativos. Este gás é inodoro, incolor e insípido, logo não detectável pelos nossos sentidos. O radão provém ainda de solos e rochas, sendo que as concentrações mais elevadas ocorrem usualmente em zonas de rochas graníticas. De acordo com a Directiva 90/143/EURATOM, nas habitações já construídas, as concentrações médias anuais não devem ultrapassar os $400 \mathrm{~Bq} / \mathrm{m}^{3}$ e nas construções futuras os níveis de radão não deverão exceder $200 \mathrm{~Bq} / \mathrm{m}^{3}$. Dinua et al. (2009) estudaram 90 habitações na

${ }^{1}$ Gás radônio é o termo utilizado no Brasil. 
Espanha cuja concentração máxima de radão era de $366 \mathrm{~Bq} / \mathrm{m}^{3}$, referindo que nessa zona havia um número excessivamente elevado de falecimentos por cancro. Chen, Rahman e Atiya (2010) analisaram as emissões de radão de alguns materiais de construção, tendo observado que um pavimento em granito com elevada emissão específica $\left(300 \mathrm{~Bq} / \mathrm{m}^{2} \mathrm{~d}\right)$ contribui somente com um valor total de $18 \mathrm{~Bq} / \mathrm{m}^{3}$, isso admitindo uma taxa de 0,3 renovação de ar por hora (Tabela 2 ).

Tem-se presente que em Portugal há muitas habitações com uma taxa RPH de apenas 0,2 , isso apesar de o valor mínimo regulamentar ser de 0,6. Para que os materiais de construção (alvenarias, bancadas de cozinha e revestimentos em granito) tenham uma quota-parte significativa em termos de emissões de radão, é assim necessário que os granitos em causa tenham uma emissão muito elevada e que a taxa de renovação de ar seja próximo de zero. Importa, contudo, ter em conta que só alguns granitos possuem elevadas emissividades específicas de radão. Chen, Rahman e Atiya (2010) referem que entre 33 variedades de granitos provenientes de vários países somente duas apresentavam emissões específicas acima de $200 \mathrm{~Bq} / \mathrm{m}^{2} \mathrm{~d}$. Já em outros países existem graves problemas relativos à utilização de materiais de construção com algum nível de radioatividade, como, por exemplo, na Suécia, onde foram contabilizadas 300.000 habitações executadas com betão à base de agregados contendo urânio, o que apresenta risco para a saúde quanto ao desenvolvimento de leucemia em crianças e jovens (AXELSON et al., 2002). Em Portugal a vigilância radiológica é uma das obrigações legais do Instituto Tecnológico e Nuclear (ITN), que regularmente procede à medições da radiação gama ambiental bem como de amostras de águas, sedimentos e peixes.

Em setembro de 2005 aquela instituição deu conta de que estudos já efetuados em 4.200 habitações permitem concluir que $60 \%$ das concentrações de radão se situam abaixo de $50 \mathrm{~Bq} / \mathrm{m}^{3}$, tendo somente $2,6 \%$ das habitações apresentado concentrações acima de $400 \mathrm{~Bq} / \mathrm{m}^{3}$. Tendo em conta que as zonas de risco com concentrações mais elevadas de radão são zonas geologicamente associadas a rochas graníticas, não é de admirar que um estudo conduzido em todo o território nacional português, como aquele levado a cabo pelo ITN, apresente valores de gravidade residual. Contudo, em 2001 a DECO já tinha publicado um estudo levado a cabo em 212 casas localizadas nos Distritos de Aveiro, Guarda e Braga (zonas com geologia fortemente granítica) que concluiu que, embora dois terços das medições tenham ficado abaixo de $200 \mathrm{~Bq} / \mathrm{m}^{3}, 22 \%$ dos casos excederam o limite de $200 \mathrm{~Bq} / \mathrm{m}^{3}$ e $17 \%$ estavam mesmo acima de $400 \mathrm{~Bq} / \mathrm{m}^{3}$ (Figura 2). Os referidos estudos não permitem, contudo, destrinçar qual a parte que diz respeito aos materiais de construção e qual a parte correspondente ao radão proveniente do solo, já que, como é evidente nas zonas de elevados afloramentos graníticos, muitas casas foram erigidas aproveitando-se essa matéria-prima, quer ao nível de alvenarias e também como revestimento de pisos.

\begin{tabular}{l|c|c|c|c|c|c}
\hline \multirow{2}{*}{ Material } & \multicolumn{3}{|c|}{ Concentração corrente $(\mathbf{B q} / \mathbf{k g})$} & \multicolumn{3}{c}{ Concentração máxima $(\mathbf{B q} / \mathbf{k g})$} \\
\cline { 2 - 7 } & ${ }^{\mathbf{2 2 6}} \mathbf{R a}$ & ${ }^{\mathbf{2 3 2}} \mathbf{T h}$ & ${ }^{\mathbf{4 0}} \mathbf{K}$ & ${ }^{\mathbf{2 2 6}} \mathbf{R a}$ & ${ }^{\mathbf{2 3 2}} \mathbf{T h}$ & ${ }^{\mathbf{4 0}} \mathbf{K}$ \\
\hline Materiais de construção & & & & & & \\
\hline Betão & 40 & 30 & 400 & 240 & 190 & 1.600 \\
\hline Betão leve & 60 & 40 & 430 & 2.600 & 190 & 1.600 \\
\hline Tijolos cerâmicos & 50 & 50 & 670 & 200 & 200 & 2.000 \\
\hline Blocos de betão & 10 & 10 & 330 & 25 & 30 & 700 \\
\hline Pedra natural & 60 & 60 & 640 & 500 & 310 & 4.000 \\
\hline Gesso natural & 10 & 10 & 80 & 70 & 100 & 200 \\
\hline Subprodutos industriais & & & & & & \\
\hline Fosfogesso & 390 & 20 & 60 & 1.100 & 160 & 300 \\
\hline Escórias & 270 & 70 & 240 & 2.100 & 340 & 1.000 \\
\hline Cinzas de carvão & 180 & 100 & 650 & 1.100 & 300 & 1.500 \\
\hline
\end{tabular}

Fonte: Kovler et al. (2002) e Kovler (2009).

Tabela 1 - Radioatividade corrente e máxima em materiais de construção e subprodutos industriais 


\begin{tabular}{l|c|c|c|c|c}
\hline \multirow{2}{*}{$\begin{array}{l}\text { Emissão específica do } \\
\text { granito }\left(\mathbf{B q} / \mathbf{m}^{\mathbf{3}} \mathbf{d}\right)\end{array}$} & \multicolumn{5}{|c}{ Taxa de renovações de ar por hora (RPH) } \\
\cline { 2 - 6 } & $\mathbf{3}$ & $\mathbf{1}$ & $\mathbf{0 , 3}$ & $\mathbf{0 , 1 5}$ & $\mathbf{0}$ \\
\hline 5 & 0,03 & 0,09 & 0,3 & 0,6 & 5 \\
\hline 10 & 0,06 & 0,2 & 0,6 & 1,2 & 25 \\
\hline 50 & 0,3 & 0,9 & 3,0 & 5,9 & 123 \\
\hline 100 & 0,6 & 1,8 & 6,0 & 12 & 246 \\
\hline 300 & 1,8 & 5,5 & 18 & 35 & 737 \\
\hline
\end{tabular}

Fonte: Chen, Rahman e Atiya (2010).

Tabela 2 - Concentração de radão em becquerel por metro cúbico emitido por piso com revestimento em granito, de acordo com o tipo de granito e com a taxa horária de renovação de ar

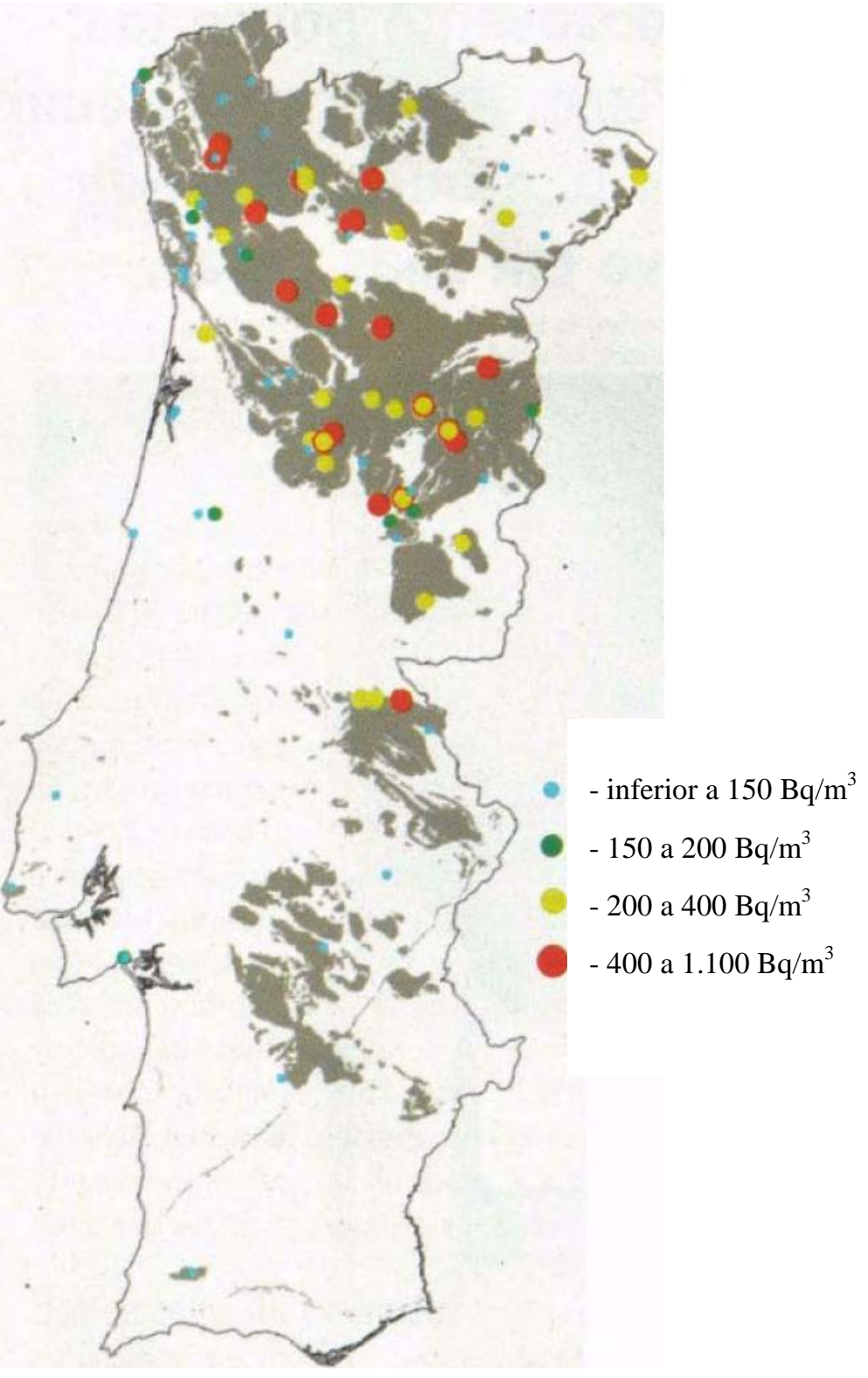

Fonte: DECO (2001).

Figura 2 - Radioatividade no interior de habitações devida ao radão 


\section{Canalizações em chumbo}

Sendo um material bastante maleável e com risco de corrosão praticamente nulo, o chumbo foi utilizado no fabrico em canalizações para abastecimento de água pelo menos desde o Império Romano (DUTRIZAC; O'REILLY; MACDONALD, 1982; HODGE, 1981; NRIAGU, 1983). Vários autores referem que o uso de canalizações para abastecimento de água feitas de chumbo provoca graves de problema de saúde, o que se fica a dever à formação de uma película de produtos de corrosão na superfície interna da tubagem, a qual acaba por ser lixiviada e contaminar a própria água (ZIETZ et al., 2009). Essa contaminação é especialmente grave em crianças e jovens, nos quais pode provocar redução de capacidades intelectuais e problemas de comportamento (CANFIELD et al., 2003; POCOCK; SMITH; BAGHURST, 1994; WILHELM; DIETER, 2003). Troesken (2006) refere vários casos de intoxicação e envenenamento por via do abastecimento de água com canalizações de chumbo durante os séculos XIX e XX, referindo que a magnitude do problema é da mesma ordem de grandeza do desastre de Chernobyl e de Bopal. Este autor refere que só nos Estados Unidos dezenas de milhares de crianças faleceram devido a esse problema e que outras tantas sofreram problemas no seu desenvolvimento cognitivo.

Tem-se presente que um teor de chumbo no sangue acima de $10 \mu \mathrm{g} / \mathrm{dl}$ se considera como valor de envenenamento (LABAT et al., 2006; TARARBIT CARRÉ; GARNIER, 2009), estando associado à mortalidade cardiovascular e ao aparecimento de cancro. Mais recentemente, Khalil et al. (2009) referem um risco acrescido de morte em pessoas idosas para concentrações de chumbo no sangue acima de $8 \mu \mathrm{g} / \mathrm{dl}$. Outros (MENKE et al., 2006) observaram risco de enfarte de miocárdio e acidente vascular cardíaco (AVC) para níveis acima de $2 \mu \mathrm{g} / \mathrm{dl}$. Embora as suspeitas sobre a possibilidade de esse material poder provocar danos irreversíveis para a saúde pública não sejam recentes, pois que já durante a década de 20 nos Estados Unidos várias entidades tenham proibido ou restringido o uso dele, essas medidas e as evidências médicas que as suportavam não foram suficientes para superar a forte oposição da indústria de produção de chumbo (RABIN, 2008). Por volta da década de 70 a própria OMS ainda admitia $300 \mu \mathrm{g} / 1$ de chumbo como o teor máximo presente na água para consumo humano. Mas de lá para cá esse valor caiu de forma abrupta (Quadro 3 ), como se os malefícios daquele metal de repente se tornassem evidentes aos olhos das entidades reguladoras.

Essa mudança de limites é de algum modo similar ao enquadramento legislativo relativo ao problema do amianto, em que se foi progressivamente admitindo o seu risco, até a proibição total da produção de materiais com amianto. Não constitui por isso admiração que o valor máximo para o teor de chumbo na água de $10 \mu \mathrm{g} / \mathrm{l}$ tenha sido objeto de um adiamento de 15 anos na Directiva 98/83/CE, pelo fato de a sua entrada em vigor implicar a substituição das canalizações em chumbo. Em Portugal a última estimativa feita, em 1995, no âmbito de um inquérito enquadrado na Directiva 98/83/CE apontava para 1.177.300 de metros de tubagem em chumbo, sendo que para a Europa esse valor rondava 16 milhões de metros de ramais e 30 milhões de metros de redes. O custo da substituição das mesmas implicava em 1999 um valor de 34.000 milhões de euros somente para a Europa com 12 países-membros (PAPADOPOULOS, 1999). Estimativas mais recentes apontam já para um valor de 200.000 milhões de euros (HAYES, 2009).

\begin{tabular}{|l|c|c|}
\hline Instrumento regulador & Ano & $\begin{array}{c}\text { Limite máximo para o teor de } \\
\text { chumbo na água }(\boldsymbol{\mu g} / \mathbf{l})\end{array}$ \\
\hline OMS & 1970 & 300 \\
\hline Directiva 80/778/CEE & 1980 & 50 \\
\hline $\begin{array}{l}\text { Directiva 98/83/CE } \\
\text { Decreto-lei no 243/2001, } \\
\text { de 5 de setembro }\end{array}$ & De 25 dez. 2003 a 25 dez. 2013 & 25 \\
\cline { 2 - 3 } & Depois de 25 de dez. 2013 & 10 \\
\hline
\end{tabular}

Quadro 3 - Evolução dos limites para o teor máximo de chumbo na água ao longo das últimas décadas 


\section{Conclusões}

A presente revisão de literatura confirma que os edifícios de habitação possuem elevadas quantidades de materiais com algum nível de toxicidade, seja durante a fase de produção, seja por libertação de substâncias tóxicas para o ar das habitações, seja pela libertação de fumos tóxicos em caso de incêndio, ou simplesmente pela contaminação da água de abastecimento público. Parte do problema está relacionada com a falta de formação de arquitetos e engenheiros nessa área, o que faz com que seja necessário ao nível acadêmico agir no sentido de corrigir tal lacuna. A verificação dos limites definidos em termos legais não é garantia suficiente de não toxicidade, por um lado, porque a questão da toxicidade dos materiais de construção tem que ver com a existência ou não de investigações que comprovem essa toxicidade, pelo que, embora algumas investigações possam não confirmar essa periculosidade para determinado material, nada obsta a que ela não se venha a confirmar. Por outro lado, porque muitos dos limites definidos na legislação em termos de toxicidade são influenciados por questões econômicas. Tudo isso sugere que a escolha de materiais num contexto de construção sustentável encare a questão da sua toxicidade como uma questão incontornável.

\section{Referências}

AGENCY FOR TOXIC SUBSTANCES AND DISEASE REGISTRY. Toxicological Profile for Creosote. Atlanta: Public Health Sector, U.S. Department of Health and Human Services, 2002.

ANTONESCU-TURCU, A.; SCHAPIRA, R. Parenchymal and Airway Diseases Caused by Asbestos. Current Opinion in Pulmonary Medicine, Philadelphia, v. 16, n. 2, p. 155-161, Mar. 2010.

AKIRA, M. Asbestosis: IPF or NSIP-Like Lesions in Asbestos-Exposed Persons, and Such Independency. Japanese Journal of Chest Diseases, Tokyo, v. 69, p. 38-44, 2010.

AXELSON, O. et al. Leukemia in Childhood and Adolescence and Exposure to Ionizing Radiation in Homes Built from Uranium-Containing Alum Shale Concrete. Epidemiology, Baltimore, v. 13, n. 2, p. 146-150, Mar. 2002.

AZUMA, K. et al. Mesothelioma Risk and Environmental Exposure to Asbestos: past and future trends in Japan. International Journal of Occupational and Environmental Health, Lodz, v. 15, n. 2, p. 166-172, Apr./June 2009.
BIANCHI, C. et al. Latency Periods in AsbestosRelated Mesothelioma of the Pleura. European Journal of Cancer Prevention, Oxford, v. 6, n. 2, p. 162-166, Apr. 1997.

CANFIELD, R. et al. Intellectual Impairment in Children with Blood Lead Concentrations below $10 \mu \mathrm{g}$ per Deciliter. New England Journal of Medicine, Boston, v. 348, n. 16, p. 1517-1526, Apr. 2003.

\section{CANUT, M. Estudo da Viabilidade do Uso do}

Resíduo Fosfogesso como Material de Construção. 2006. 154 f. Dissertação (Mestrado em Engenharia) - Escola de Engenharia, Universidade Federal de Minas Gerais, Belo Horizonte, 2006.

CHEN, J.; RAHMAN, N.; ATIYA, I. Radon Exhalation from Building Materials for Decorative Use. Journal of Environmental Radioactivity, Essex, v. 101, n. 4, p. 317-322, Apr. 2010.

CRUZ, H.; NUNES,L. Durabilidade e Protecção de Estruturas de Madeira. Construção Magazine, Porto, n. 34, p. 36-38, 2009

DECO. Radão: perigo que se esconde no granito. Proteste, Lisboa, n. 241, p. 37-39, nov. 2001.

DINUA et al. Lung Cancer Attributable to Indoor Radon Exposures in Two Radon: Prone Areas, Ştei (Romania) and Torrelodones (Spain). AIP Conference Proceedings, New York, v. 1131, p. 175-180, May 2009.

DOROUDIANI, S.; OMIDIAN, H.

Environmental, Health and Safety Concerns of Decorative Mouldings made of Expanded Polystyrene in Buildings. Building and Environment, Oxford, v. 45, n. 3, p. 647-654, Mar. 2010.

DUTRIZAC, J.; O’REILLY, J.; MACDONALD, R. Roman Lead Plumbing: did it really contribute to the decline and fall of the empire. CIM Bulletin, Montreal, v. 75, n. 841, p. 111-115, 1982.

EDLICH, R.; WINTERS, K.; LONG, W. Treated Wood Preservatives linked to Aquatic Damage, Human Illness, and Death: a societal problem. Journal of Long-Term Effects of Medical Implants, New York, v. 15, n. 2, p. 209-223, 2005.

EUROPEAN ATOMIC ENERGY COMMUNITY. Council Directive 96/29 EC. Luxembourg, 1996. 
FLORES, A. et al. Organoclorados: um problema de saúde pública. Ambiente \& Sociedade, Campinas, v. 7, n. 2, p. 111-124, 2004.

GANN, R. et al. Fire Conditions for Smoke Toxicity Measurements. Fire Materials, London, v. 18, n. 3, p. 193-199, 1994.

HALL, J.; HARWOOD, B. Smoke or Burns: which is deadlier? National Fire Protection Association Journal, Boston, v. 38, p. 38-43, 1995.

HANSEN, S.; BURROUGHS, H. Classifying Indoor Air Problems: managing indoor air quality. Lilburn: Fairmont, 1999.

HAUSER, R.; CALAFAT, A. Phthalates and Human Health. Occupational and

Environmental Medicine, London, v. 62, n. 11, p. 806-818, 2005

HAYES, C. Plumbo Solvency Control: best practice guide. London: IWA Specialista Group on Metals and Related Substances in Drinking Water, 2009.

HEUDORF, U. et al. Phthalates: toxicology and exposure. International Journal of Hygiene and Environmental Health, Jena, v. 210, n. 5, p. 623634, Oct. 2007.

HODGE, A. Vitrivius, Lead Pipes and Lead Poisoning. American Journal of Archaeology, New York, v. 85, n. 4, p. 486-491, Oct. 1981.

INTERNATIONAL AGENCY FOR RESEARCH ON CANCER. Polychlorinated Dibenzo-paraDioxins and Polychlorinated Dibenzofurans. Lyon, 1997. (IARC Monographs on the Evaluation of Carcinogenic Risks to Humans, 69).

INTERNATIONAL AGENCY FOR RESEARCH ON CANCER. Wood Dust and Formaldehyde. Lyon, 1995. (IARC Monographs on the Evaluation of Carcinogenic Risks to Humans, 62).

\section{INTERNATIONAL COMMISSION ON}

RADIOLOGICAL PROTECTION.

Recommendations of the International Commission on Radiological Protection. Oxford: Pergamon, 1990. (ICRP Publication, 60).

JARVHOLM, B.; ENGLUND, A.; ALBIN, M. Pleural Mesothelioma in Sweden: an analysis of the incidence according to the use of asbestos. Occupational and Environmental Medicine, London, v. 56, n. 2, p. 110-113, Feb. 1999.
KHALIL, N. et al. Association of Blood Lead Concentrations with Mortality in Older Women: a prospective cohort study. Environmental Health, London, v.8, p. 15, Apr. 2009.

KOOPMAN-ESSEBOOM, C. et al. Effects of Polychlorinated Biphenyl/Dioxin Exposure and Feeding Type on Infants' Mental and Psychomotor Development. Pediatrics, Evanston, v. 97, n. 5, p. 700-706, May 1996.

KOSTIAINEN, R. Volatile Organic Compounds in the Indoor Air of Normal and Sick Houses. Atmospheric Environment, Oxford, v. 29, n. 6, p. 693-702, 1995.

KOVLER, K. Radiological Constraints of Using Building Materials and Industrial By-Products in Construction. Construction and Building Materials, Guildford, v. 23, n. 1, p. 264-253, Jan. 2009.

KOVLER, K. et al. Natural Radionuclides in Building Materials Available in Israel. Building and Environment, Oxford, v. 37, n. 5, p. 531-537, May 2002.

KWOK, N. et al. Substrate Effects on VOC Emissions from an Interior Finishing Varnish. Building and Environment, Oxford, v. 38, n. 8, p. 1019-1026, Aug. 2003.

LABAT, L. et al. Variabilité de la Mesure de la Plombémie Pour de Faibles Concentrations Proches du Seuil de $100 \mu \mathrm{g} / \mathrm{l}$ : étude multicentrique. Annales de Toxicologie Analytique, Poitiers, v. 18, n. 4, p. 297-304, 2006.

LADOU, J. The Asbestos Cancer Epidemic. Environmental Health Perspectives, Research Triangle Park, v. 112, n. 3, p. 285-290, 2004.

LANTING, C. et al. Neurologic Condition in 42Month-Old Children in Relation to Pre-and Postnatal Exposure to Polychlorinated Biphenyls and Dioxins. Early Human Development, Amsterdam, v. 50, n. 3, p. 700-706, Feb. 1998.

LEVIN,B.; KULIGOWSKI,E. Toxicology of Fire and Smoke. In: SALEM, H.; KATZ, S. (Ed.). Inhalation Toxicology. New York: CRC, 2005. p. 205-228.

LIANG, H.; HO, M. Toxicity Characteristics of Commercially Manufactured Insulation Materials for Building Applications in Taiwan.

Construction and Building Materials, Guildford, v. 21, n. 6, p. 1254-1261, June 2007. 
LOVEKAMP-SWAN T.; DAVIS, B. Mechanisms of Phthalate Ester Toxicity in the Female Reproductive System. Environmental Health Perspectives, Research Triangle Park, v. 111, n. 2, p. 139-145, Feb. 2003.

MEEKER, J. et al. Urinary Phthalate Metabolites in Relation to Preterm Birth in Mexico City. Environmental Health Perspectives, Research Triangle Park, v. 117, n. 10, p. 1587-1592, Oct. 2009.

MENKE, A. et al. Blood Lead below 0,48 $\mu \mathrm{mol} / \mathrm{l}$ $(10 \mu \mathrm{g} / \mathrm{dl})$ and Mortality among US Adults. Circulation, Baltimore, v. 114, n. 13, p. 13881394, Sept. 2006.

NRIAGU, J. Saturnine Gout among Roman Aristocrats: did lead poisoning contribute to the fall of the empire? New England Journal of Medicine, Boston, v. 308, n. 11, p. 660-663, Mar. 1983.

OPPENHUIZEN, A.; SIJM, D. Bioaccumulation and Biotransformation of Polychlorinated Dibenzo-p-Dioxins and Dibenzofurans in Fish. Environmental Toxicology and Chemistry, New York, v. 9, n. 2, p. 175-186, Feb. 1990.

PAPADOPOULOS, I. Revision of the Council Directive on the Quality of Water Intended for Human Consumption. The Environmentalist, Hampshire, v. 19, n. 1, p. 23-26, 1999.

PAPAEFTHYMIOU, H.; GOUSETI, O. Natural Radioactivity and Associated Radiation Hazards in Building Materials Used in Peloponnese, Greece. Radiation Measurements, Oxford, v. 43, n. 8, p. 1453-1457, Sept. 2008.

POCOCK, S.; SMITH, M.; BAGHURST, P. Environmental Lead and Children's Intelligence: a systematic review of the epidemiological evidence. British Medical Journal, London, v. 309, n. 6963, p. 1189-1197, Nov. 1994.

PORTUGAL. Decreto-Lei no 181 . Diário da República, Lisboa, $1^{a}$ série, n. 172, p. 6578-6583, 06 set. 2006.

PORTUGAL. Decreto-Lei n ${ }^{\circ} 266$. Diário da República, $1^{a}$ série, n. 141, p.4689-4696, 24 jul. 2007a.

PORTUGAL. Decreto-Lei no 284. Diário da República, Lisboa, $1^{a}$ série, n. 194, p. 3540-3548, 24 ago. 1989.

PORTUGAL. Despacho no 17.141. Diário da República, Lisboa, $2^{a}$ série, n. 149, p. 2215322514, 03 ago. 2007 b.
PORTUGAL. Diretiva 83/477/CEE. Protecção Sanitária dos Trabalhadores Expostos ao Amianto durante o Trabalho. Jornal Oficial, Lisboa, n. L206, p. 0016-0018, 29 jul. 1991a.

PORTUGAL. Diretiva 90/143/EURATOM. Recomendação da Comissão, de 21 de Fevereiro de 1990, relativa à Protecção da População contra a Exposição Interior ao Radão. Jornal Oficial, Lisboa, n. L080, p. 0026-0028, 27 mar. 1990.

PORTUGAL. Diretiva 91/382/EEC. Altera a Directiva 83/477/CEE, relativa à Protecção Sanitária dos Trabalhadores Expostos ao Amianto durante o Trabalho. Jornal Oficial, Lisboa, $n$. L206, p. 0016-0018, 29 jul. 1991b.

PORTUGAL. Diretiva 98/83/CE. Qualidade da Água Destinada ao Consumo Humano. Jornal Oficial, Lisboa, n. L330, 03 nov. 1998.

PORTUGAL. Diretiva 204/42/CE. Relativa à Limitação das Emissões de Compostos Orgânicos Voláteis (COV) Resultantes da Utilização de Solventes Orgânicos em Certas Tintas e Vernizes e Produtos de Revestimento Automóvel. Jornal Oficial, Lisboa, n. 143, 21 abr. 2004.

PORTUGAL. Diretiva 2003/18/EC. Protecção Sanitária dos Trabalhadores Expostos ao Amianto durante o Trabalho. Jornal Oficial, Lisboa, n. 97, 15 abr. 2003.

PRUSZINSKI, A. Review of the Landfill Disposal Risks and the Potential for Recovery and Recycling of Preservative Treated Timber. Adelaide: Environmental Protection Authority, 1999.

RABIN, R. The Lead Industry and Lead Water Pipes “A Modest Campaign". American Journal of Public Health, Boston, v. 98, n. 9, p. 15841592, Sept. 2008.

RIHANEK, S. T. Radioactivity of Phosphate Plaster and Phosphate Gypsum. Tonindustrie Zeitung, Berlin, v. 95, n. 9, p. 264-270, 1971.

SALASAR, C. Estudo sobre Emissão de Compostos Orgânicos Voláteis COVS em Tintas Imobiliárias à Base de Solvente e Água. 2007. 83 f. Dissertação (Mestrado em Química) Universidade Estadual de Londrina, 2007.

SAMFIELD, M. Indoor Air Quality Data Base for Organic Compounds. Research Triangle Park: Air and Energy Engineering Research Laboratory, U.S. Environmental Protection Agency, 1992. 
SATYANAND, T. Aftermath of the Bhopal Accident. The Lancet, London, v. 371, n. 9628, p. 1900, June 2008.

SILVERSTEIN, M.; WELCH, L.; LEMEN, R. Developments in Asbestos Cancer Risk Assessment. American Journal of Industrial Medicine, New York, v. 52, n. 11, p. 850-858, Nov. 2009.

SMITH, P. Risks to Human Health and Estuarine Ecology Posed by Pulling Out Creosote-Treated Timber on Oyster Farms. Aquatic Toxicology, Amsterdam, v. 86, n. 2, p. 287-298, Jan. 2008.

STERLING, D. Volatile Organic Compounds in Indoor Air: an overview of sources, concentrations, and health effects. In: GAMMAGE, R.; JACOBS, V. (Ed.). Indoor Air and Human Health. Chelsea: Lewis, 1985. p. 387.

SWAN, S. Environmental Phthalate Exposure in Relation to Reproductive Outcomes and Other Health Endpoints in Humans. Environmental Research, San Diego, v. 108, n. 2, p. 177-184, Oct. 2008.

TARARBIT, K.; CARRÉ, N.; GARNIER, R. Occurrence of Lead Poisoning during Follow-Up of Children at Risk with Initial Screening Lead Blood Levels Below $100 \mu \mathrm{g} / \mathrm{l}$. Revue d'Epidemiologie et de Sante Publique, Paris, v. 57, n. 4, p. 249-255, Aug. 2009.

TILLITT, D. et al. Dioxin-Like Toxic Potency in Forster's Tern Eggs from Green Bay, Lake Michigan, North America. Chemosphere, Oxford, v. 26, n. 11, p. 2079-2084, June 1993.

THIERFELDER, T.; SANDSTROM, E. The Creosote Content of Used Railway Crossties as Compared with European Stipulations for Hazardous Waste. Science of the Total Environment, Amsterdam, v. 402, n. 1, p. 106112, Aug. 2008.
THORTON, J. Environmental Impacts of Polyvinyl Chloride (PVC) Building Materials. Washington, DC: Healthy Buiding Network, 2002.

TROESKEN, W. The Great Lead Water Pipe Disaster. Cambridge: MIT Press, 2006.

UNITED NATIONS CENTRE FOR HUMAN SETTLEMENTS. Building Materials and Health. Nairobi, 1997.

\section{UNITED STATES ENVIRONMENTAL} PROTECTION AGENCY. Potential Uses of Phosphogypsum and Associated Risks. Washington, DC, 1992.

VARMA, R.; MULAY, S. The Bhopal Accident and Methyl Isocyanate Toxicity. In: GUPTA, R. C. (Ed.). Toxicology of Organophosphate \& Carbamate Compounds. Amsterdam: Elsevier, 2006. p. 79-88.

WILHELM, M.; DIETER, H. Lead Exposure via Drinking Water: unnecessary and preventable. Umweltmedizin in Forschung und Praxis,v. 8, p. 239-241, 2003.

WOLFF, M. et al. Prenatal Phenol and Phthalate Exposures and Birth Outcomes. Environmental Health Perspectives, Research Triangle Park, v. 116, n. 8, p. 1092-1097, Aug. 2008.

WU, C. Discussion on Fire Safety Factors from Case Studies of Building Fires. 2001. 190 f. Tese (Mestrado em Engenharia Civil) - University of Tainan, Taiwan, 2001.

ZIETZ, B. et al. Lead Pollution of Drinking Water in Lower Saxony from Corrosion of Pipe Materials. Gesundheitswesen, Berlin, v. 71, n. 5, p. 265-274, May 2009. 